\title{
A Novel Deep Learning Pipeline Architecture based on CNN to Detect Covid-19 in Chest X-ray Images
}

\author{
Putra Sumari $^{1}$, Saqib Jamal Syed ${ }^{1}$, Laith Abualigah ${ }^{2 *}$ \\ ${ }^{1}$ School of Computer Science, Universiti Sains Malaysia, Penang, Malaysia \\ putras@usm.my,saqib@usm.my \\ ${ }^{2}$ Faculty of Computer Sciences and Informatics, Amman Arab University, Amman 11953, Jordan \\ Aligah.2020@gmail.com \\ *Corresponding author: Laith Abualigah (Aligah.2020@gmail.com)
}

Article History: Received: 10 November 2020; Revised 12 January 2021 Accepted: 27 January 2021; Published online: 5 April 2021

\begin{abstract}
Covid-19 is a severe public health problem worldwide. To date, it has spanned worldwide, with 24.6 million infected with 835,843 confirm the death. Covid-19 detection is indeed an important task and has to be done as quickly as possible so that treatment and monitoring can be carried out early. The current world standard RT-PCR screening for Covid-19 detection has to cope with the world population's great demand. There is a need to have an alternative way to cope with the demands. It has to be a quick and accurate detection procedure, such as using a chest x-ray for Covid-19 detection. This paper proposes a deep learning pipeline architecture called Gray Level Co-occurrence Matrix GLCM) with Convolutional Neural Network (CNN) for Covid-19 detection using chest X-ray image. The proposed method has two main diagnosis features, a quicker diagnosis, and a detailed diagnosis. The quicker diagnosis uses few GLCM features and a standard neural network (NN) algorithm to detect Covid-19 symptoms. It is a suitable method for rural areas where computing resources are minimal. The detailed diagnosis uses huge image pixel features and a deep convolutional neural network (CNN) algorithm to detect Covid19 symptoms. It is a suitable method for places where computing resources are sufficient. The proposed work provides the highest classification performance, with $97.06 \%$ accuracy compared to other similar works.
\end{abstract}

Keywords: Deep learning; Covid-19; Chest X-ray image; Convolutional neural network; GLCM.

\section{INTRODUCTION}

Covid-19 is a pandemic to the world today. It becomes a serious public health problem and has been declared a global health emergency worldwide (Roosa et al., 2020). The pandemic is started in March 2020 and now has spanned all over the world with a total of 24.6 million cases with 835,843 confirm the death. The number of cases is rapidly increased since it is spread through human to human transmission (Li et al., 2020). The traveler seems the most easily people can be affected. In many developed countries, the Covid-19 has caused panic due to insufficient screening agents to cater to the increasing number of people. Seeing this, it is very critical to have a quicker detection of Covid-19 as many people as possible and yet to minimize the impact quickly (Kowalski et al., 2020).

The diagnosis of Covid-19 begins whenever a person is having signs of infection, such as respiratory symptoms, fever, cough, and dyspnea. Then the diagnosis follows by a real-time reverse transcription-polymerase chain reaction (RT-PCR) procedure for the final appearance of Covid-19. However, the world standard RT-PCR is low sensitivity, and it requires many days to get the result (Ai et al., 2020). Today, the number of candidates and demands increases, the RT-PCR agents seem insufficient to cater more inspection to more peoples. As a result, they may not receive appropriate treatment quickly. Meantime, chest X-ray images are proven as a useful diagnostic method in most of today's medical applications (Ardila et al., 2019). The use of chest X-ray images for detecting Covid-19 has advantages in two ways (Al-Qaness, Ewees, Fan, Abualigah, \& Abd Elaziz, 2020).

First, it is a faster, easier, cheaper, and less harmful method than a CT scan. Second, it compliments the RT-PCR method in detecting more people quickly. However, the problem of detecting the appearance of Covid-19 objects in digital X-ray images is quite tricky. The problems are in many ways. First, the chest X-ray is a grayscale format; high sensitivity, soft tissue, and low contrast are hard to analyze. The object, such as nodules, military opacities, and pleural effusion, are hard to identify. Figure 1 shows an example of (a) healthy chest, whereas (b) infected one in which the appearance, structure, and distribution of objects are difficult to distinguish between them (Xu et al., 2020). 


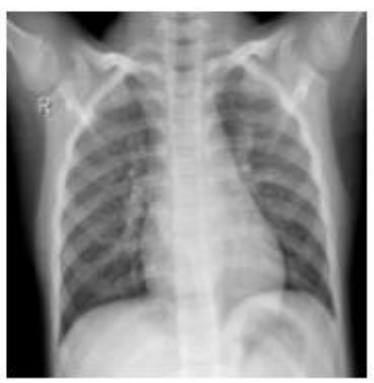

(a) Health X-ray

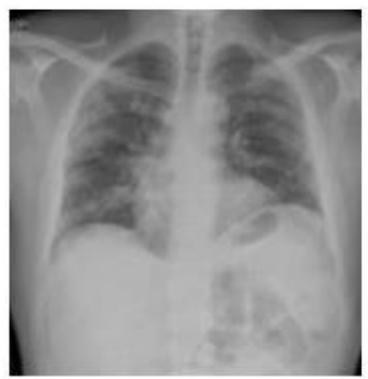

(b) Covid-19 positive

Fig. 1. (a) Health chest X-ray mage and (b) Positive Covid-19 Chest X-ray images.

Today, neural network (aka deep learning) is becoming a pivotal role. It has proven to be a useful tool for building most machine learning applications. Computer vision can recognize almost a thousand objects, draw art, drive a car, and many more. In medical applications, it able to assist the doctor in diagnosing cancer and detecting tumor cells (Şahan, Polat, Kodaz, \& Güneş, 2007). However, deep learning models often need a lot of experimental tuning, high processing power, and colossal dataset to operate. Delicate tuning and limited datasets are the main difficulties faced by researchers. Getting the best dataset is crucial, and yet to annotate them is a most very cumbersome task.

In respect of the grayscale issue of chest X-ray images, limited dataset, lack processing power, and excessive tuning in deep learning, in this study, we propose a deep learning architecture called gray level co-occurrence matrix (GLCM) with convolution neural network (CNN) for Covid-19 detection based on the chest X-ray image. The main contributions of this paper are given as follows. 1- End-to-end deep learning model pipeline architecture is proposed for Covid-19 detection based on chest X-ray. 2- We show that the combination of GLCM and deep learning is a useful model for analyzing grayscale chest X-ray image issue. 3- Chest X-ray images are the best tool for the detection of Covid-19. The proposed model has been yield very high results in the small dataset (147 Covid-19 vs.130 Normal). The proposed method illustrated promising results in detecting the Covid-19 virus.

The paper is organized as follows: the literature review is elaborated in section 2. The detail of the proposed work called GLCMCNN pipeline architecture for covid-19 detection is presented in section 3. The experimental and results are elaborated in section 4. Finally, the conclusion is summarized in section 5.

\section{BACKGROUND AND LITERATURE SURVEY}

\subsection{Brain Neuron inspired Deep learning}

The idea of deep learning technology is derived from human brain biological systems (Barrett, Morcos, \& Macke, 2019). The main elements in the brain system are neurons, as shown in figure 1.3a. A neuron consists of the axon, cell body, and dendrites. The neuron is an excitable electrochemical cell that uses synapse connections to receive signals or stimuli from other neurons (figure 2). A neuron's cell body may have many dendrites and just one axon. The cell body receives impulses that are expressed via the dendrites. If a stimulus is strong enough, the neuron produces a second stimulus with different firing rates transmitted through synapses along the axon to the next neuron. Biological neurons form millions of connections in this way, creating a three-dimensional neural network. These connections and the term firing let us humans have dreams, memories, ideas, self-regulated movement, reflexes, and others.
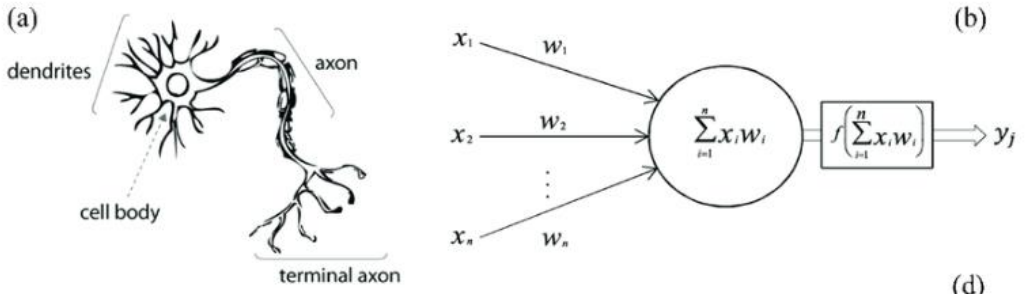

(c)
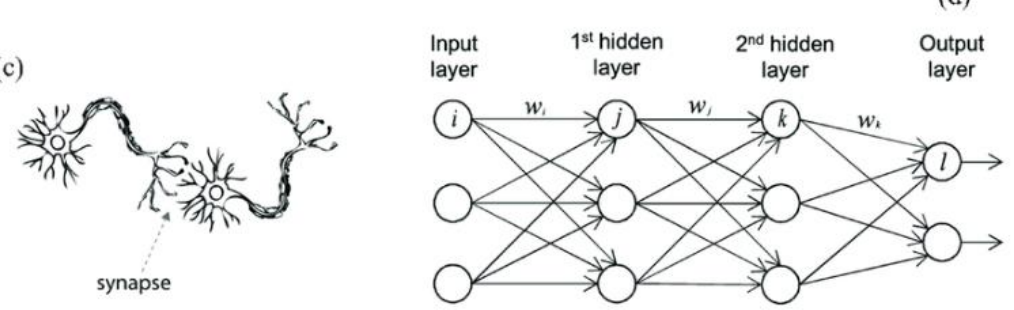

Fig. 2. A biological neuron in comparison to an artificial neural network. (a) Brain neuron, (b) Artificial neuron, (c) Neuron \& biological synapse, (d) Artificial neural network 
In computational modeling (Landin \& Rowe, 2013), the neuron is referred to as perceptron or nodes (fig. 2b). The interconnection of millions of brain neurons is a multilayer neural network (fig.2d). Fig $2 \mathrm{~d}$ shows nodes in layer view (first hidden layer and second hidden layer) with input and output. Node is an individual processing element, as shown in fig. 2 (b). As for biological neurons, they receive stimuli from other neurons, so artificial neurons (nodes) receive data inputs ( $\mathrm{x} 1$, $\mathrm{x} 2, . . \mathrm{xn}$ ). Data input is processed, and output is produced at each node. The result is forwarded to the next node. Weights (w) and transfer mechanism are terms used to describe the strength of connections between artificial neurons (node). They imitated the neural network's learning by several feed-forward and back forward through a supervised learning paradigm. The weights and biases are keep changing, controlled by learning rate along with optimizer function. Multilayer neural networks can have thousands of multiple layers between the input and the output. Having thousands or millions of layers yields high processing power in performing learning tasks in deep learning applications.

\subsection{Convolutional Neural Network (CNN)}

A Convolutional Neural Network $(\mathrm{CNN})$ is the foundation of most image-based artificial intelligent applications. The building block of $\mathrm{CNN}$ is shown in figure 3. It has two main components, the convolutional and the fully neural network (connected layers). The convolution is extracting and reducing an image into its essential features. The fully neural network is the learning and understanding of the image (Aslan, Unlersen, Sabanci, \& Durdu, 2021). Other optimization methods can be used to tune the CNN such as Arithmetic Optimization Algorithm (AOA) (Abualigah, Diabat, Mirjalili, Abd Elaziz, \& Gandomi, 2021) and Aquila Optimizer (AO) (Abualigah, Yousri, et al., 2021).

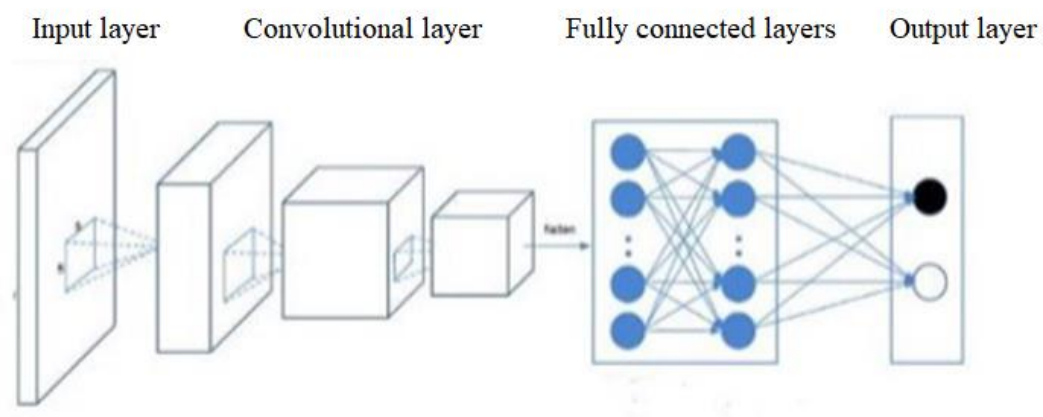

Figure 3: The architecture of convolutional neural network.

\subsection{Literature Survey}

Deep learning or neural network is a rising technology for artificial intelligence (AI) in recent years. The neural network is an intelligent classification algorithm proven to be accurate in medical imaging applications (Suzuki, 2017) significantly to help the doctor minimize errors during diagnosis. The neural network gives better accuracy in diagnosing and segmenting lung cancer (Al-Tarawneh, 2012; Manikandarajan \& Sasikala, 2013; Yousri et al., 2021), good performance in classifying skin lesions (Esteva et al., 2017), better accuracy in predicting and recognizing meaningful breast cancer patterns (Şahan et al., 2007).

In all these works, the neural network is applied to CT scans and X-ray images. The CT scan and X-ray images can show and differentiate body components such as fractures, bone dislocations, lung infections, and tumors. The CT scan and X-ray images also show a soft structure of the active body part and inner soft tissues and organs (Şahan et al., 2007). In a new Covid-19 domain, only just recently, researchers use neural networks to predict and recognize the meaningful pattern of Covid-19. It begins mostly in the laboratory with a CT Scan. To differentiate Covid-19 pneumonia and Influenza-A from safe cases, researchers used an early neural network screening model (Shan et al., 2020). (Şahan et al., 2007) describes a study that uses a deep learning method to extract the graphical features of Covid-19 to provide a clinical diagnosis before pathogenic testing, saving valuable time for disease diagnosis. Covid-19, known as MERS-CoV, is diagnosed by a neural network found in (Xu et al., 2020).

A neural network also diagnoses another cousin of Covid-19 known as SARS-CoV is found in (Gozes et al., 2020; Wang et al., 2021). X-ray images are a quicker, simpler, less expensive, and less dangerous alternative to a CT scan (Fang et al., 2020). Still, not many diagnoses work to establish with X-ray images along with the neural network method. The earlier work on Covid-19 using deep learning with chest X-ray images are in (Chen \& Su, 2018; Mukherjee et al., 2021). These works, however, are facing a short dataset.

\section{THE PROPOSED GLCM-CNN PIPELINE ARCHITECTURE}

The proposed GLCM-CNN pipeline architecture for covid19 detection based on chest X-ray images is shown in Figure 4. The architecture has two main components (shown in the dotted box). The first component (top dotted box) is called the 
GLCMneural network component for quick detection of Covid-19, and a second component (bottom dotted box) is called the pixel-CNN component for precise detection of Covid-19.

The first component uses a few gray level co-occurrence matrix (GLCM) chest X-ray features to feed to the primary standard neural network(NN) model. It is designed for a quick and fast diagnosis of Covid19. Few GLCM features and necessary NN lead to low processing power machine and suitable for a rural area where computer resources are minimal. The second component uses huge pixel value features of chest X-ray to feed to a deep convolutional neural network (CNN) model. It is designed for a detailed diagnosis of Covid-19. CNN is known to be a heavy processing power. Therefore it is designed for high performance and suitable for hospitals where resources are well equipped. The proposed architecture gives the developer a choice of design to use for Covid-19 detection. The second component can be skipped in the rural area with resources at minimal.

The overall process of Figure 4 starts with chest X-ray images is processed by extracting four GLCM texture features known as contrast, homogeneity, correlation, and energy. Then, these four features are fed to the neural network model for a quick diagnosis. The output is produced, which is either positive Covid19 or normal X-ray. The positive Covid-19 X-ray can then be passed to the RT-PCR process for further treatment. The normal X-ray can then be diagnosed once again through the second component (pixel-CNN method). The normal X-ray is transformed into pixel-based features. Then, the pixel features are fed to the convolutional neural network for detailed diagnosis. Finally, the output is produced, which is either positive Covid-19 or normal X-ray. The positive covid-19 X-ray can be passed to the RT-PCR process for further treatment.

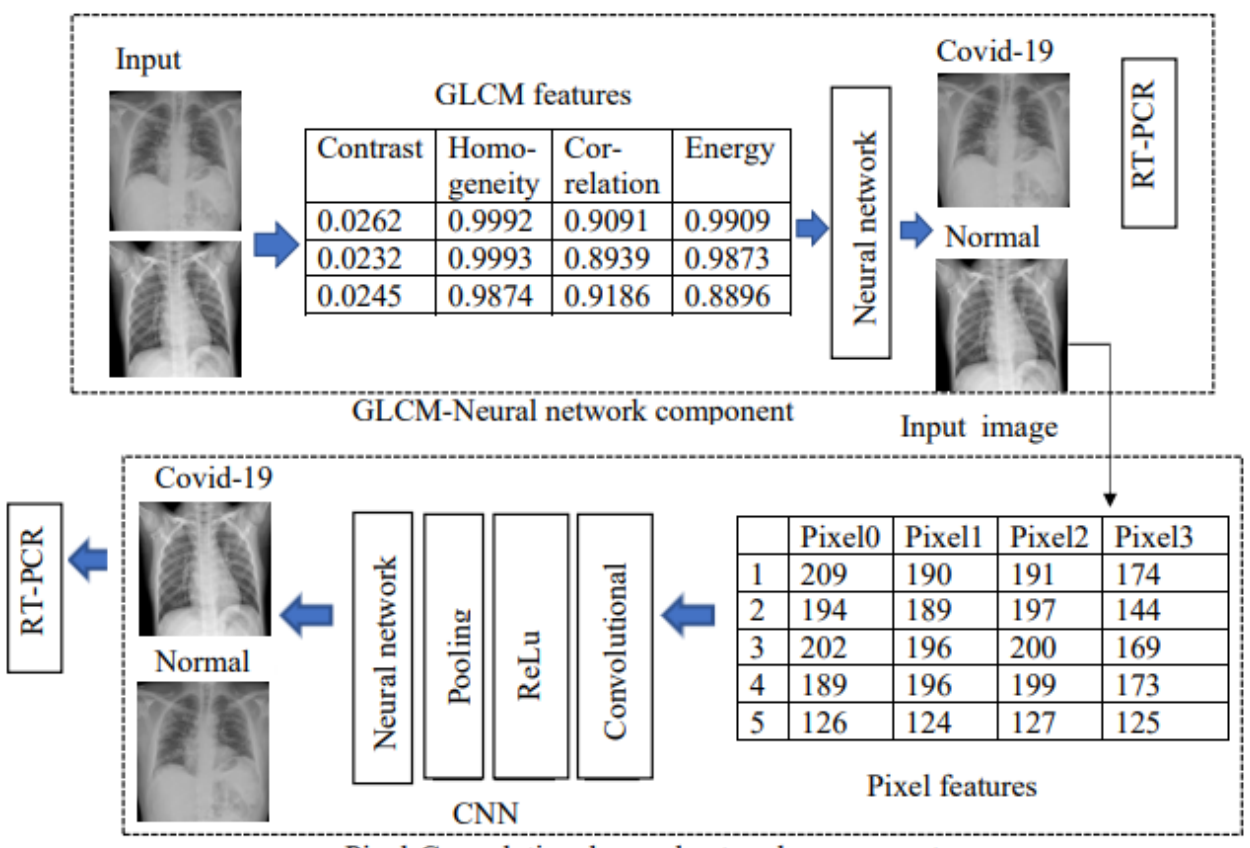

Pixel-Convolutional neural network component

Fig. 4. The overall GLCM-CNN pipeline architecture for Covid-19 detection based on chest X-ray image.

\subsection{GLCM Neural Network component}

The system consists of two main components, the GLCM extraction process, and neural network learning components, as shown in Figure 5. The GLCM component extracts four types of X-ray images' texture properties to be fed into the neural network layers. The neural network layer learns and analyses those features and classify them into either positive covid-19 xray or normal X-ray. The primary motivation was to create a light architecture with a small number of parameters (weights) in order to avoid long computation times. The proposed GLCM-NN component architecture's minimal parameters are computationally efficient and can prevent overfitting. Due to the heavy use of parameters and longer training time, deep architectures are more susceptible to overfitting. As a result, the proposed work is best suited to mass population screening, especially in resource-strapped areas. 


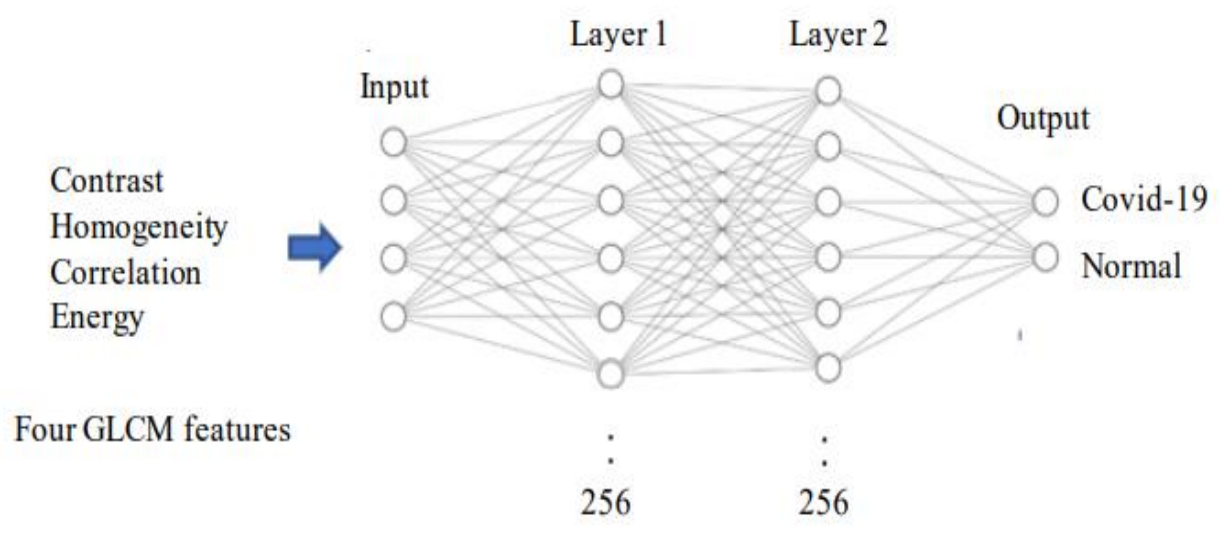

Fig. 5. GLCM-Neural network architecture.

\subsubsection{GLCM Feature extraction}

Gray Level Co-occurrence Matrix (GLCM) is a matrix representing texture features information of chest X-ray image. The texture feature is represented by four metrics; contrast, homogeneity, correlation, and energy. Figure 6 shows an example of four features of four chest X-rays. These four features represent the best texture of the chest X-ray images. They have been successfully proven the best textures in representing image properties in many medical diagnosis applications. These four features are calculated by taking into account the spatial relationship between two pixels at a given distance and angle of orientation (orientation angle $0,45,90,135$ degrees). As a characteristic matrix function, GLCM forms a matrix concurrence (joint occurrence) of the image. These four texture properties of GLCM define as follows (Suzuki, 2017).

- In GLCM, energy represents the gray level concentration of intensity. In GLCM, it returns the number of squared components. $\sum_{i, j=1}^{M} P_{i, j}^{2}$ is used to define it. In a horizontal and vertical matrix, I and $\mathrm{j}$ are the coordinates, and $P i j$ is the matrix meaning in the $i$ and $j$ coordinate.

- Contrast measures the intensity contrast between a pixel and its neighbour over the whole image. It is define by the following $\sum_{i, j=1}^{M} P_{i, j}(i-j)^{2}$. In a horizontal and vertical matrix, $i$ and $j$ are the coordinates, and $P i j$ is the matrix meaning in $i$ and $j$ coordinate.

- Homogeneity is a measure of how similar the elements in the GLCM are to the diagonal. The opposite of the contrast value is the homogeneity value. Define by $\sum_{i, j=1}^{M} P_{i, j} /\left(1+(i-j)^{2}\right)$. In a horizontal and vertical matrix, $i$ and $j$ are the coordinates, and $P i j$ is the matrix meaning in the $i$ and $j$ coordinate.

- Homogeneity measures closeness distribution of elements in the GLCM to the GLCM diagonal. Homogeneity values are the inverse of contrast value. Define by $\sum_{i, j=1}^{M} P_{i, j} /\left(1+(i-j)^{2}\right)$. The $i$ and $j$ are the coordinates in horizontal and vertical matrix and $P_{i j}$ is matrix value in $i$ and $j$ coordinate.

- Correlation measures the correlated pixel to its neighbour over the entire image. Define by $\sum_{i, j=1}^{M} P_{i, j}\left[\frac{\left(i-\mu_{i}\right)\left(i-\mu_{i}\right)}{\sqrt{\left(\sigma_{i}^{2}\right)}\left(\sigma_{i j}^{2}\right)}\right]$. In a horizontal and vertical matrix, $i$ and $j$ are the coordinates, and $P i j$ is the matrix meaning in the $i$ and $j$ coordinate.

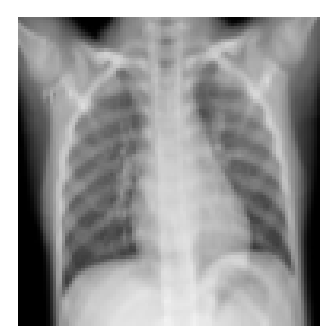

$\Rightarrow$\begin{tabular}{|l|l|l|l|}
\hline Contrast & Homogeneity & Correlation & Energy \\
\hline 0.0262 & 0.9992 & 0.9091 & 0.9909 \\
\hline 0.0232 & 0.9993 & 0.8939 & 0.9873 \\
\hline 0.0245 & 0.9874 & 0.9186 & 0.8896 \\
\hline 0.0225 & 0.9932 & 0.9845 & 0.9979 \\
\hline
\end{tabular}

Figure 6. Four features are extracted from X-ray image.

\subsubsection{Neural network}

The neural network model consists of input layers, two hidden layers, and an output layer shown in Figure 3 . The input layer consists of four neurons as a placeholder for four GLCM texture features of the chest X-ray image. The two hidden layers consist of 256 nodes each, as it is considered very low computation in many deep learning applications. The output layer consists of two neurons as classes, either positive Covid-19 or normal chest X-ray. The weight used random distribution; the 
activation function used rectified linear function (ReLu). The output layer used the softmax function. Other parameters such as chest X-ray image size and batch size will be varied during the experiment.

\subsection{Pixel-CNN component $\mathrm{x}$}

The system consists of two-man components; pixel features extraction component and convolutional neural network (CNN) component, as shown in Figure 7. The input is a matrix form of the whole chest X-ray image. Each entry in the matrix is a pixel value. These pixel values are then sent to convolutional neural network layers for learning purposes. The output is either positive Covid-19 or standard X-ray images. The positive Covid-19 will go through the RT-PCR process for treatment, and the standard chest X-rays are healthy.

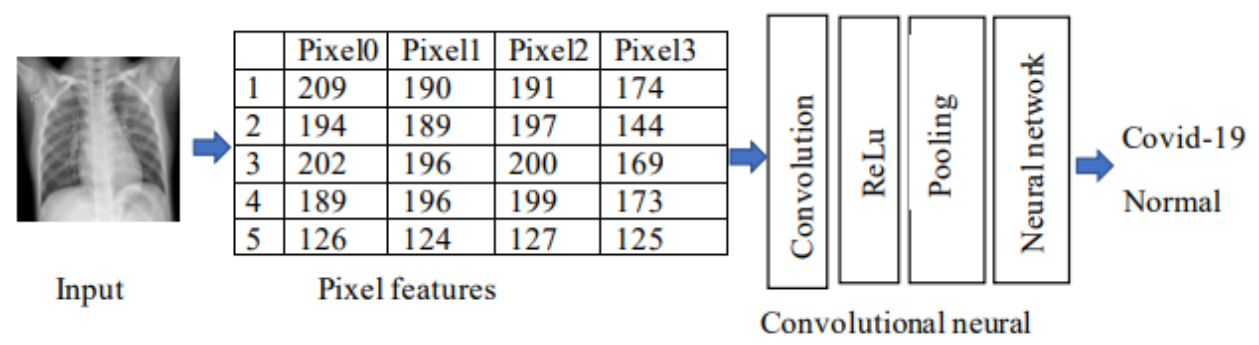

Fig. 7. Pixel-convolutional neural network (CNN) architecture.

\subsubsection{Pixel features extraction.}

Each X-ray image is scaled into $\mathrm{m} \times \mathrm{n}$ format and will be transformed into matrix $1 \times(m * n)$; each entry in the matrix contains pixel value as shown in Figure 8. The case of $K$ sample in the dataset, then it yields matrix $(K \times(m * n))$. Matrix $(K$ $\times(m * n))$ is in the form of CSV file and Figure 6 shows $K=5$. All pixel values in the matrix will be normalized into a value in range between $0-1$. This a CSV file is then become the input to the CNN component for learning purposes.

\begin{tabular}{|l|l|l|l|l|l|l|}
\hline & Pixel0 & Pixel1 & Pixel2 & Pixel3 & Label \\
\hline 1 & 209 & 190 & 191 & 174 & Normal \\
\hline 2 & 194 & 189 & 197 & 144 & Covid-19 \\
\hline 3 & 202 & 196 & 200 & 169 & Normal \\
\hline 4 & 189 & 196 & 199 & 173 & Normal \\
\hline 5 & 126 & 124 & 127 & 125 & Covid-19 \\
\hline
\end{tabular}

Input Pixel features are extracted from X-ray images

Fig. 8. Pixel features of 5 chest X-ray images are presented in CSV file.

\subsubsection{Convolutional neural network component}

As shown in Figure 9, the convolutional neural network model consists of the input layer, the convolutional layers, the ReLu layer, the pooling layer, the flatten layer, the neural network layer, and the output layer. The input layer is a matrix of size $\mathrm{K}$ $\times(m * n) . K$ is the number of chest X-ray images. A few filters then convolute the input image. The main component of this architecture is the convolutional layer, which senses the presence of a series of features in the input. A group of convolutional kernels make up this layer. This layer's operation can be calculated as follows:

$$
f_{c}^{k}(m, n)=\sum_{d} \sum_{r, s} j_{d}(r, s) \cdot 1_{c}^{k}(v, W) .
$$

where $j_{d}(r, s)$ is $n$ the input vector's instance $j_{d}$, which is multiply by $l_{c}^{k}(v, w)$ index of the $k^{\text {th }}$ kernel of the $c^{\text {th }}$ layer. The $k t h$ kernel's output mapping can be calculated as:

$f_{c}^{k}\left[f_{c}^{k}(1,1) \ldots, f_{c}^{k}(m, n), \ldots, f_{c}^{k}(M, N)\right]$.

The pooling layer is placed between two convolutional layers, which reduce the size of the vectors while maintaining their relevance. It collects all of the relevant data in the receptive domain's area. It uses the feedback within that region to generate output $Y_{c}^{k}=0_{p}\left(F_{c}^{k}\right) . Y^{k}{ }_{c}$ determines the pooled feature map of the $c^{\text {th }}$ layer for the $k^{\text {th }}$ kernel, and $0 \mathrm{p}$ determines the type of pooling procedure to be performed. The convolution layer parameter is set with Rectified Linear Unit (ReLU) for activation function, Adam for the optimizer, Softmax for output activation function, and max pooling for pooling filter. The output of the max-pooling filter is flattened and send to the neural network layer. The neural network uses two hidden layers with 256 
neurons each. Finally, two outputs are produced. An essential hyper-parameters of convolutional neural network values such as image size, the filter number, filter size, pooling window size, and batch size will be tested.

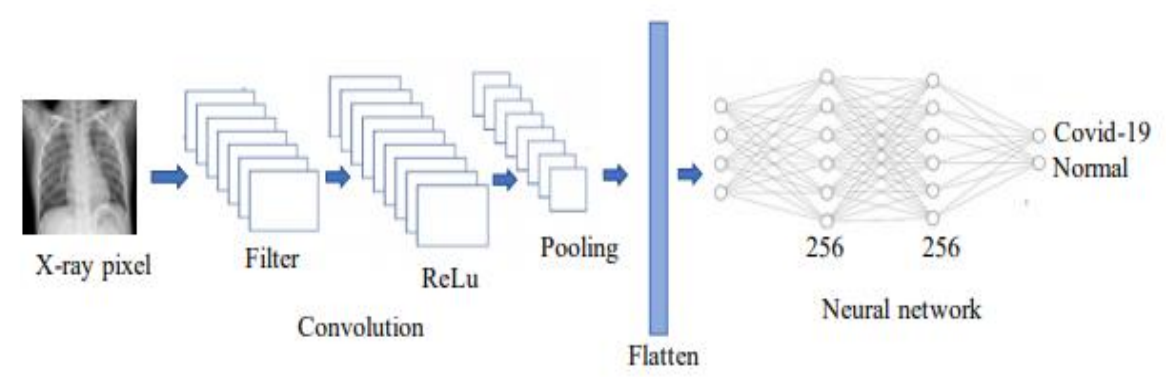

Figure 9. Convolutional neural network architecture.

\section{EXPERIMENTAL AND RESUTS}

\subsection{Dataset}

Dataset is quite a balance which consists of 143 positive Covid-19 X-ray and 130 negative Covid-19 X-ray (normal), which total is 273 X-rays frontal view. The 143 Covid-19 X-ray positives are taken from (Loey, Smarandache, \& M Khalifa, 2020) and the 130 negative covid-19 X-ray (normal) are taken from (Khan, Shah, \& Bhat, 2020). All images in this dataset were initialized 50 x 50 pixels in size.

\subsection{Hardware and software}

Python programming language (specifically Tensor flow and Keras libraries) was used to train the proposed work. All of the tests were carried out on a Google Colaboratory Linux server running Ubuntu 16.04 and a Tesla K80 GPU graphics card.

\subsection{Experimental set up}

The model is experimental with parameters as in table 1 . The batch size, input image size, number of filters, size filter and pooling window size, were experimentally set in table 1 where the rest of the parameter remains constant. The dataset was divided into two separate datasets at random, with 80 percent and $20 \%$ used for training and research, respectively.

Table 1. Parameters and its values

\begin{tabular}{|l|l|}
\hline Parameters & Values \\
\hline Weight & Random \\
\hline Activation function & ReLu \\
\hline Out layer & Softmax \\
\hline Optimizer & Adam \\
\hline Epoch & 100 \\
\hline Batch Size & $25,50,75,100,125,150$ \\
\hline Input Image Size & $50,50,100 \times 150 \times 150$ \\
\hline
\end{tabular}

\begin{tabular}{|l|l|}
\hline NN set up & Values \\
\hline Layer & 2 \\
\hline Nodes per layer & 256 \\
\hline Class & 2 \\
\hline
\end{tabular}




\begin{tabular}{|l|l|}
\hline CNN set up & Values \\
\hline ConVlayer & 1 \\
\hline Filter & $5,10,20,30,40$ \\
\hline Size filter & $2 \times 2,3 \times 3,4 \times 4,5 \times 5,6 \times 6$ \\
\hline Nodes per layer & 256 \\
\hline Pooling window size & $2 \times 2,3 \times 3,4 \times 4$ \\
\hline Class & 2 \\
\hline
\end{tabular}

4.4 Performance metrics

Accuracy $=(\mathrm{TN}+\mathrm{TP})=(\mathrm{TN}+\mathrm{TP}+\mathrm{FN}+\mathrm{FP})$ and uncertainty matrix were used as success parameters. The letters TP, FP, TN, and FN, stand for True Positive, False Positive, True Negative, and False Negative. Given a test dataset and model, TP is the proportion of positive (Covid-19) that the model correctly labels as Covid-19; FP is the proportion of negative (normal) that the model incorrectly labels as positive (Covid-19); TN is the proportion of negative (normal) that is correctly labeled as normal, and FN is the proportion of positive (Covid-19) that the model incorrectly labels as negative (normal).

\section{TUNING RESULT}

For all experiments, 5-fold cross-validation was used to verify the proposed architecture. The proposed work deals with a convolutional neural network $(\mathrm{CNN})$ in which requires several parameters. Therefore, the first experiments were based on finding the best parameters. As shown in Table 1, a few essential parameters were considered, including batch size, input image size, number of filters, size filter, and pooling window size. The result is as follows.

\subsection{Input image size}

Different sizes of chest X-ray images were included in the dataset. They've been scaled down to a xed dimension. The resized dimensions were set to 50x50, 100x 100, and 150x 150 pixels for testing purposes. The proposed model produced a better result (accuracy $=97.06 \%$ ) from a chest X-ray of size $100 \times 100$ as compared to 50x50 (accuracy $=95.23 \%$ ) and $150 \times 150($ accuracy $=60 \%)$.

\subsection{Batch size}

Various batch sizes were used during the training phase, varying from 25 to 150 instances with a difference of 25 , as shown in table 1. As shown in table 2, the best result was obtained from both 50 and 75 cases, with an accuracy of 97.06 percent. 147 COVID-19 positive cases were correctly reported in both batch sizes. Table 2 shows the extensive experimental test results for various batch sizes.

\subsection{Filter Number in $C N N$}

Different numbers of filters were employed in the convolution layer, 5, 10, 20, and 30. and 40. It is observed that the best results were obtained from the experimental test, where 30 filters were used.

\subsection{Filter Size}

The scale of the convolution filter was also experimented with, like $2 \times 2,3 \times 3, \ldots, 6 \times 6$. The filter of size $5 \times 5$ provided the best performance, with an accuracy of 97.06 percent.

5.5 Pooling Window Size

The convolutional pooling window size was experimental with $2 \times 2,3 \times 3$, and $4 \times 4$. The best result was obtained from the pooing window size $2 \times 2$ with accuracy $=97.06 \%$.

Table 2. Confusion matrix of different batches.

\begin{tabular}{|c|c|c|}
\hline \multicolumn{2}{|c}{ Batch size $=25$, Accuracy $=94.33 \%$} \\
\hline & Covid-19 & Non Covid-19 \\
\hline Covid-19 & 144 & 3 \\
\hline Non Covid-19 & 11 & 136 \\
\hline
\end{tabular}

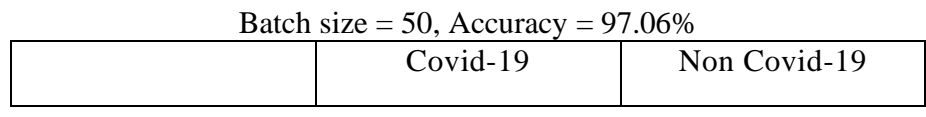




\begin{tabular}{|c|c|c|}
\hline Covid-19 & 147 & 0 \\
\hline Non Covid-19 & 6 & 141 \\
\hline
\end{tabular}

\begin{tabular}{|c|c|c|}
\hline \multicolumn{3}{|c}{ Batch size $=75$, Accuracy $=97.06 \%$} \\
\hline & Covid-19 & Non Covid-19 \\
\hline Covid-19 & 147 & 0 \\
\hline Non Covid-19 & 6 & 141 \\
\hline
\end{tabular}

\begin{tabular}{|c|c|c|}
\hline \multicolumn{3}{|c|}{ Batch size $=100$, Accuracy $=92.12 \%$} \\
\hline & Covid-19 & Non Covid-19 \\
\hline Covid-19 & 139 & 8 \\
\hline Non Covid-19 & 10 & 137 \\
\hline
\end{tabular}

\begin{tabular}{|c|c|c|}
\hline \multicolumn{3}{|c}{ Batch size $=125$, Accuracy $=95.11 \%$} \\
\hline & Covid-19 & Non Covid-19 \\
\hline Covid-19 & 145 & 2 \\
\hline Non Covid-19 & 11 & 136 \\
\hline
\end{tabular}

\begin{tabular}{|c|c|c|}
\hline \multicolumn{2}{|c}{ Batch size $=150$, Accuracy $=89.22 \%$} \\
\hline & Covid-19 & Non Covid-19 \\
\hline Covid-19 & 132 & 15 \\
\hline Non Covid-19 & 25 & 122 \\
\hline
\end{tabular}

\section{PERFORMANCE COMPARISON}

The same set of experimental datasets was applied to other common deep learning (DL) architectures for comparison, such as shallow CNN (Mukherjee et al., 2021), MobileNet (Chen \& Su, 2018) and VGG16 (Alippi, Disabato, \& Roveri, 2018). In this experiment we used 30 filters of size $5 \times 5$ each, $2 \times 2$ pooling window size, 50 and 75 batch size, and 100x100 input image size. Their performance scores were presented in Table 3. The proposed GLCM-CNN based architecture outperformed MobileNet, VGG and Shallow CNN by more than $16.83 \%, 94.12 \%$ and $0.5 \%$ in terms of accuracy respectively. Not only did the proposed model outperform in terms of precision, but it also outperformed in terms of false negative and false positive.

Table 3. Performance comparison with other deep learning models

\begin{tabular}{|l|l|l|l|l|}
\hline Metric & $\begin{array}{l}\text { MobileNet (Chen } \\
\text { \& Su, 2018) }\end{array}$ & $\begin{array}{l}\text { VGG (Alippi et al., } \\
\mathbf{2 0 1 8})\end{array}$ & $\begin{array}{l}\text { Shallow } \\
\text { (Mukherjee et al., } \\
\mathbf{2 0 2 1})\end{array}$ & GLCM-CNN \\
\hline False Positive rate & 0.1161 & 0.5000 & 0.000 & 0.0000 \\
\hline False negative rate & 0.2095 & 0.000 & 0.0580 & 0.0100 \\
\hline Accuracy (\%) & 83.08 & 50.00 & 96.92 & 97.06 \\
\hline
\end{tabular}

\section{CONCLUSION}

In this paper, a GLCM-CNN pipeline architecture is introduced to idintyfy Covid-19 positive cases. The detection is based on Chest X-ray images. The proposed work is designed to suit rural areas where resources are limited, and for hospitals where resources are efficient. The GLCM-NN combination is used for quick diagnosis in places where resources are limited. The pixel-CNN combination is used for detailed diagnosis in places where resources are sufficient. The experiments are condcuted and valiadted on dataset collections of Covid-19 positive and healthy Chest X-rays. To validate its robustness, a 5 -fold cross-validation protocol was conducted. The proposed work achieved the best accuracy at $97.06 \%$ with a set of the following parameters: 100x100 input image size, 50 and 75 batch size, 30 filters of size $5 \times 5$ each, and $2 \times 2$ pooling window 
size. A comparison study was also performed against state-of-the-art works such as MobileNet, VGG-16, and Shallow CNN. Our work improved by more than $16.83 \%, 94.12 \%$, and $0.5 \%$ inaccuracy, respectively. The proposed model outperformed in terms of false-negative and false-positive rates.

\section{REFERENCES}

[1]. Abualigah, L., Diabat, A., Mirjalili, S., Abd Elaziz, M., \& Gandomi, A. H. (2021). The arithmetic optimization algorithm. Computer Methods in Applied Mechanics and Engineering, 376, 113609.

[2]. Abualigah, L., Yousri, D., Abd Elaziz, M., Ewees, A. A., Al-qaness, M. A., \& Gandomi, A. H. (2021). Aquila Optimizer: A novel meta-heuristic optimization Algorithm. Computers \& Industrial Engineering, 107250.

[3]. Ai, T., Yang, Z., Hou, H., Zhan, C., Chen, C., Lv, W., . . Xia, L. (2020). Correlation of chest CT and RTPCR testing for coronavirus disease 2019 (COVID-19) in China: a report of 1014 cases. Radiology, 296(2), E32-E40.

[4]. Al-Qaness, M. A., Ewees, A. A., Fan, H., Abualigah, L., \& Abd Elaziz, M. (2020). Marine predators algorithm for forecasting confirmed cases of COVID-19 in Italy, USA, Iran and Korea. International journal of environmental research and public health, 17(10), 3520.

[5]. Al-Tarawneh, M. S. (2012). Lung cancer detection using image processing techniques. Leonardo Electronic Journal of Practices and Technologies, 11(21), 147-158.

[6]. Alippi, C., Disabato, S., \& Roveri, M. (2018). Moving convolutional neural networks to embedded systems: the alexnet and VGG-16 case. Paper presented at the 2018 17th ACM/IEEE International Conference on Information Processing in Sensor Networks (IPSN).

[7]. $\quad$ Ardila, D., Kiraly, A. P., Bharadwaj, S., Choi, B., Reicher, J. J., Peng, L., . . Corrado, G. (2019). End-toend lung cancer screening with three-dimensional deep learning on low-dose chest computed tomography. Nature medicine, 25(6), 954-961.

[8]. Aslan, M. F., Unlersen, M. F., Sabanci, K., \& Durdu, A. (2021). CNN-based transfer learning-BiLSTM network: A novel approach for COVID-19 infection detection. Applied Soft Computing, 98, 106912.

[9]. Barrett, D. G., Morcos, A. S., \& Macke, J. H. (2019). Analyzing biological and artificial neural networks: challenges with opportunities for synergy? Current opinion in neurobiology, 55, 55-64.

[10]. Chen, H.-Y., \& Su, C.-Y. (2018). An enhanced hybrid MobileNet. Paper presented at the 20189 th International Conference on Awareness Science and Technology (iCAST).

[11]. Esteva, A., Kuprel, B., Novoa, R. A., Ko, J., Swetter, S. M., Blau, H. M., \& Thrun, S. (2017). Dermatologist-level classification of skin cancer with deep neural networks. nature, 542(7639), 115-118.

[12]. Fang, Y., Zhang, H., Xie, J., Lin, M., Ying, L., Pang, P., \& Ji, W. (2020). Sensitivity of chest CT for COVID-19: comparison to RT-PCR. Radiology, 296(2), E115-E117.

[13]. Gozes, O., Frid-Adar, M., Greenspan, H., Browning, P. D., Zhang, H., Ji, W., . . Siegel, E. (2020). Rapid ai development cycle for the coronavirus (covid-19) pandemic: Initial results for automated detection \& patient monitoring using deep learning ct image analysis. arXiv preprint arXiv:2003.05037.

[14]. Khan, A. I., Shah, J. L., \& Bhat, M. M. (2020). CoroNet: A deep neural network for detection and diagnosis of COVID-19 from chest X-ray images. Computer Methods and Programs in Biomedicine, 196, 105581.

[15]. Kowalski, L. P., Sanabria, A., Ridge, J. A., Ng, W. T., de Bree, R., Rinaldo, A., ... Bradford, C. R. (2020). COVID - 19 pandemic: effects and evidence - based recommendations for otolaryngology and head and neck surgery practice. Head \& neck, 42(6), 1259-1267.

[16]. Landin, M., \& Rowe, R. C. (2013). Artificial neural networks technology to model, understand, and optimize drug formulations Formulation Tools for Pharmaceutical Development (pp. 7-37): Elsevier.

[17]. Li, Q., Guan, X., Wu, P., Wang, X., Zhou, L., Tong, Y., . . . Wong, J. Y. (2020). Early transmission dynamics in Wuhan, China, of novel coronavirus-infected pneumonia. New England journal of medicine.

[18]. Loey, M., Smarandache, F., \& M Khalifa, N. E. (2020). Within the lack of chest COVID-19 X-ray dataset: a novel detection model based on GAN and deep transfer learning. Symmetry, 12(4), 651.

[19]. Manikandarajan, A., \& Sasikala, S. (2013). Detection and Segmentation of Lymph Nodes for Lung Cancer Diagnosis. Paper presented at the National Conference on System Design and Information Processing.

[20]. Mukherjee, H., Ghosh, S., Dhar, A., Obaidullah, S. M., Santosh, K., \& Roy, K. (2021). Shallow convolutional neural network for COVID-19 outbreak screening using chest X-rays. Cognitive Computation, 1-14.

[21]. Roosa, K., Lee, Y., Luo, R., Kirpich, A., Rothenberg, R., Hyman, J., . . Chowell, G. (2020). Real-time forecasts of the COVID-19 epidemic in China from February 5 th to February 24th, 2020. Infectious Disease Modelling, 5, 256-263.

[22]. Şahan, S., Polat, K., Kodaz, H., \& Güneş, S. (2007). A new hybrid method based on fuzzy-artificial immune system and k-nn algorithm for breast cancer diagnosis. Computers in Biology and Medicine, $37(3), 415-423$.

[23]. Shan, F., Gao, Y., Wang, J., Shi, W., Shi, N., Han, M., ... Shi, Y. (2020). Lung infection quantification of COVID-19 in CT images with deep learning. arXiv preprint arXiv:2003.04655.

[24]. Suzuki, K. (2017). Overview of deep learning in medical imaging. Radiological physics and technology, $10(3), 257-273$. 
[25]. Wang, S., Kang, B., Ma, J., Zeng, X., Xiao, M., Guo, J., . . Meng, X. (2021). A deep learning algorithm using CT images to screen for Corona Virus Disease (COVID-19). European Radiology, 1-9.

[26]. Xu, X., Jiang, X., Ma, C., Du, P., Li, X., Lv, S., . . Su, J. (2020). A deep learning system to screen novel coronavirus disease 2019 pneumonia. Engineering, 6(10), 1122-1129.

[27]. Yousri, D., Abd Elaziz, M., Abualigah, L., Oliva, D., Al-Qaness, M. A., \& Ewees, A. A. (2021). COVID-19 X-ray images classification based on enhanced fractional-order cuckoo search optimizer using heavytailed distributions. Applied Soft Computing, 101, 107052. 